\title{
An Exactly Solvable Model of a Multidimensional Incommensurate Structure
}

\author{
A. L. Figotin and L. A. Pastur \\ Institute for Low Temperature Physics and Engineering, UkrSSR Academy of Sciences, Kharkov, \\ USSR
}

\begin{abstract}
The paper considers the class of Schrödinger multidimensional discrete operators with quasi-periodic unbounded potential for which essentially complete spectral analysis may be carried out. In the case of sufficiently high incommensurability of almost-periods, the spectrum of such operators is found to be pure point and simple, the eigenfunctions exponentially localized and the low frequency conductivity exponentially small. In the one-dimensional case, for any incommensurability, the spectrum does not contain the absolutely continuous component, while for small incommensurability the spectrum is singular continuous.
\end{abstract}

\section{Introduction}

The spectral properties of differential and finite difference operators with almost periodic coefficients raise at present considerable interest. The reason is both a very wide range of their potential applications and the variety of types of spectral behaviour that depends on the coefficients forms and the arithmetic properties of almost periods. In terms of the theory of disordered systems, almost periodic operators, which model in the one-body approximation the so-called incommensurate structures, are in an intermediate position between completely ordered systems, represented in the same approximation by equations with periodic coefficients and completely disordered systems for which the respective coefficients are random functions with sufficiently good mixing properties. Whereas in these two cases the structures and behaviours of basic physical quantities may be regarded in quite a number of interesting cases as clear enough (at least qualitatively or on the theoretical physics level of rigour [8]), understanding of the incommensurate system is now living through its formation (cf., e.g., refs. 1 and 13).

This paper, whose principal results were announced in ref. 11, studies the structure of the spectrum and estimates the low frequency conductivity of the incommensurate structure model described by a family of Hamiltonians $H_{d}(\omega)$ 
of the form:

$$
\left[H_{d}(\omega) \psi\right]_{x}=\sum_{y \in \mathbb{Z}^{d}} w_{x-y} \psi_{y}+g \tan \alpha_{x} \psi_{x}
$$

where $w_{x}$ is a sequence of complex numbers satisfying the conditions

$$
w_{x}=w_{x}^{*}, \quad\left|w_{x}\right| \leqq C e^{-\rho|x|}, \quad C, \rho>0
$$

and

$$
\alpha_{x}=\pi(\alpha, x)-\omega, \alpha \in \mathbb{R}^{d}, \omega \in(0, \pi),(\alpha, x)=\sum_{j=1}^{d} \alpha_{j} x_{j} .
$$

The model (1.1)-(1.3) is the natural generalization for the case of arbitrary dimension $d>1$ of the remarkable one-dimensional model proposed by Fishman, Grempel and Prange [4] in which, under the condition that an irrational number $\alpha$ is not well approximated by rationals, essentially all the eigenfunctions are explicitly found which prove to be exponentially localized and the corresponding spectrum is shown to be a dense set in $\mathbb{R}$. However the method of ref. [4] is rather indirect and complicated even in the one-dimensional case. We shall show that a simple and essentially algebraic procedure can yield a convenient representation of the resolvent of the operator $H_{d}$ (see Eq. (2.4)) to be used in a detailed spectral analysis of the operator.

Now we summarize the basic results. In order to avoid some merely technical complications arising from the second term in Eq. (1.1) going to infinity, we shall assume that ${ }^{1}$

$$
\omega \neq \frac{\pi}{2}-\pi(\alpha, x)(\bmod \pi), x \in \mathbb{R}^{d}
$$

Theorem 2. Let a frequency vector $\alpha \in \mathbb{R}^{d}$ satisfy the following diophantine condition:

$$
|(\alpha, x)-m| \geqq C_{1}|x|^{-\beta}, C_{1}, \beta>0, m \in \mathbb{R}, x \in \mathbb{R}^{d} .
$$

Then for any $\omega$ for which Eq. (1.4) is valid, the spectrum of the operator $H_{d}$ is pure point, simple (nondegenerate) ${ }^{2}$, dense in $\mathbb{R}$, and all the eigenfunctions decay exponentially when $|x| \rightarrow \infty$.

Theorem 3. Let $\sigma(v)$ be the conductivity of the system at zero temperature in an oscillating electric field of the frequency $v$ (for the definition see Sect. 5). Then for $\nu \downarrow 0$ and $\alpha$, satisfying condition (1.5),

$$
\sigma(v) \leqq C_{2} \exp \left(-C_{3} v^{-1 / \beta}\right),
$$

where the constant $C_{3}$ is of the same order of magnitude as the constant $C_{1}$ in condition (1.5).

1 Most of our results remain valid even when the condition is not observed; however in this case it is only the sequences going to zero in the poles of $\tan \alpha_{x}$ that will enter into the domain of the operator (1.1)

2 There is one eigenfunction corresponding to each $x \in \mathbb{Z}^{d}$ (localized in the neighbourhood of $x$ ) 
Theorems 2 and 3 show that the spectrum of $H_{d}$, for any spectral parameter value, is strongly localized, which in case of disordered systems is commonly presumed to take place only in the vicinity of fluctuation boundaries [8]. This seems natural in the one-dimensional case $(d=1)$ considered in ref. [4], because the "potential" $g \tan (\pi n \alpha+\omega)$ takes on, under condition (1.4), arbitrarily large values on a sequence of points going to infinity ${ }^{3}$. It is however interesting that these peaks are also rather thick and irregular under condition (1.5) in the multidimensional case, so that a particle, no matter how large its energy is, still is "entangled" in them and cannot escape to infinity.

Theorem 2, together with some additional considerations, provides rather a detailed and complete description of the dependence of the spectrum of the operator $H_{1}$ on the arithmetic properties of $\alpha$.

In case of rational $\alpha$, i.e. periodical "potential",

$$
q_{x}=g \tan \alpha_{x}, \quad \alpha_{x}=\pi \alpha x+\omega, \quad x \in \mathbb{R},
$$

the spectrum of operator (1.1)-(1.4) for $d=1$ is, as is well known, absolutely continuous. In case of nearest neighbour interaction, i.e. of the second order equation, where

$$
w_{x}=0, \quad x \neq \pm 1
$$

the spectrum has multiplicity two.

If, on the contrary, $\alpha$ is irrational, there is no absolutely continuous component in the spectrum $H_{1}$. This statement might be proved by the method proposed in ref. 9 based on positivity of the Lyapunov exponent. In the case under consideration this positiveness follows from equality of the densities of states of operator (1.1)-(1.4) and of the Lloyd model [cf. ref. 8], the Thouless formula [10, 13, 15] relating the density of states to the Lyapunov exponent and positivity of the Lyapunov exponent in the Lloyd model. However the statement of ref. 9 and the Thouless formula have by now been only proved for second order equations and for the case that the "potential" $q_{x}$ has at least a finite first moment $\left(\lim _{N \rightarrow \infty} N^{-1} \sum_{|x| \leqq N}\left|q_{x}\right|<\infty\right)$; therefore this proof of the absence of the absolutely continuous spectrum is strictly speaking inapplicable to operators of the form involved. Therefore in the Appendix we present a proof of the absence of an absolutely continuous component using the modification of the technique used to prove Theorems $1-3$, for operators (1.1)-(1.4), without assuming validity of condition (1.5):

Theorem 4. Let $d=1$ and $\alpha$ be any irrational number. Then the spectrum of operator (1.1)-(1.4) does not contain an absolutely continuous component, i.e. is singular.

Note also that if the spectrum $H_{1}$ is under condition (1.8) singular, it has multiplicity one. This statement (whose proof will be presented elsewhere) is general in nature and follows from an extension to the discrete case of the theorem

3 In terms of the theory of almost periodic functions, $\tan (\pi n \alpha+\omega)$ is a Levitan $(N)$ almost periodic function, rather than a Bohr almost periodic function (uniform) [7] 
of I. S. Kac [6] which states that the spectrum of a second order operator on the whole axis has multiplicity one, if the Lebesgue measure of the intersection of absolutely continuous spectra components of the Neumann problem on both the semiaxes is zero.

Further, if $\alpha$ is irrational and is not well approximated by rationals, then, according to Theorem 2, the spectrum of the operator $H_{1}$ is pure point and the eigenfunctions decay exponentially. If on the other hand the number $\alpha$ approximation by rationals proves good enough, so that there exists a sequence of rational numbers $p_{n} / q_{n}$ satisfying the inequality

$$
\left|\alpha-p_{n} / q_{n}\right| \leqq \text { Const } n^{-q_{n}}
$$

then the spectrum of the operator $H_{1}$, under the additional condition $(1.8)^{4}$, is pure singular continuous and the generalized eigenfunctions do not tend to zero when $|x| \rightarrow \infty$. This can be proved both on the basis of the A. Ya. Gordon theorem [5, 13], as was done to the almost Mathieu equation [13], and by the technique we shall develop and use in this paper.

J. Bellissard et al. [2] considered the operators of the form of (1.1) and even more general ones, with the periodic function as $q_{x}(1.7)$, having a pole in every period, monotonically increasing between poles and can be meromorphically continued into a certain strip. By using the KAM theory, the authors proved validity of the statement similar to Theorem 2 under the additional condition of smallness of the off-diagonal part $W$ of the operator $H_{d}$. Our results seem to provide reasons to think that the latter condition has no close relation to the essence of the problem and that the existence of poles in $V(\zeta)$ is probably sufficient for localization of a particle with any energy.

In our case, by using the modified perturbation theory method similar to that used in ref. 2 , one can show that Theorem 2 remains valid when the following term is added to operator (1.1):

$$
\varepsilon V(\exp [2 \pi i(\alpha, x)+2 i \omega]) \psi_{x},
$$

where $V(z), z \in \mathbb{C}$ is a function analytic in the vicinity of the unit circle $|z|=1$, mapping it into the real axis, and $\varepsilon$ is sufficiently small (cf. Theorem $2^{\prime}$ in Sect. 3).

Note also that existence of multidimensional finite difference operators with an almost periodic and even a limit periodic potential, having a pure point spectrum was proved by Craig [3] and Pöschl [12]. Unlike ref. 2 and this paper, refs. 3 and 12 considered bounded potentials, and the spectrum closure in this case may be either a Cantor set of zero measure or a whole interval. However the method used there to construct the operator with the specified spectrum (inverse spectral method) and based on the perturbation theory (KAM theory) cannot yield the explicit form of the potential and with necessity implies a small off-diagonal part.

To close this section we would like to point out that B. Simon and J. Avron private communication) found independently and by other methods results similar to ours: completeness of eigenfunctions of ref. 4 , multidimensional generali-

4 It is sufficient to assume that the function $W_{x}$ has finite support 
zation, the phenomena that occur when the frequencies $\alpha$ have good diophantine properties.

\section{General Formalism and Density of States}

Denote by $W$ the operator in $\ell_{2}\left(\mathbb{Z}^{d}\right)$ specified by the first term in (1.1), and by $U$ the unitary operator of multiplication by $\exp [2 \pi i(\alpha, x)]$. Then, by virtue of relation $\tan z=-i\left(1-e^{2 i z}\right) /\left(1+e^{2 i z}\right)$ we may write that when $\operatorname{Im} z \neq 0$,

$$
\begin{aligned}
H_{d}-z= & (W-z-i g)\left[I+(W-z+i g)(W-z-i g)^{-1} e^{2 i \omega} U\right] \\
& \times\left(I+e^{2 i \omega} U\right)^{-1} .
\end{aligned}
$$

Hence, assuming that for $\operatorname{Im} z \neq 0$,

$$
B=(W-z-i g)^{-1}, C=-(W-z+i g)(W-z-i g)^{-1}, \varkappa=e^{2 i \omega},
$$

we find that the resolvent $R(z)=\left(H_{d}-z\right)^{-1}$ of the operator $H_{d}$ from (1.1) may be represented as

$$
R(z)=(I+\varkappa U)(I-\varkappa C U)^{-1} B .
$$

Since for $\operatorname{Im} z \neq 0\|C\|<1$, then this representation suggests that $R(z)$ may be written as the following series uniformly divergent when $\operatorname{Im} z \neq 0$ :

$$
R(z)=\sum_{n=0}^{\infty}\left(I+e^{2 i \omega} U\right)(C U)^{n} B e^{2 i \omega n}=B-2 i g \sum_{n=1}^{\infty} B(U C)^{n-1} U B e^{2 i \omega n} .
$$

Below we shall need the Fourier transformation transferring vectors $\Psi_{x}$ from $\ell_{2}\left(\mathbb{Z}^{d}\right)$ into square-integrable functions $\Psi(\eta)$ on a $d$-dimensional torus $T_{d}=$ $\left\{\eta: \eta=\left(\eta_{1}, \ldots, \eta_{d}\right), \mid \eta_{j} \cdot=1\right\}$

$$
\begin{aligned}
\Psi(\eta) & =\sum_{x \in \mathbb{Z}^{d}} \psi_{x} \eta^{x}, \eta^{x}=\eta_{1}^{x_{1}} \ldots \eta_{d}^{x_{d}} \\
\psi_{x} & =(2 \pi i)^{-d} \int_{T^{d}} \Psi(\eta) \eta^{x-1} d \eta .
\end{aligned}
$$

By this transformation the finite difference operators $W, B, C$ of (1.1) and (2.2) are converted into operators of multiplication by functions

$$
\begin{aligned}
& W(\eta)=\sum_{x \in \mathbb{Z}^{d}} w_{x} \eta^{x}, B(\eta)=(W(\eta)-z-i g)^{-1}, \\
& C(\eta)=-(W(\eta)-z+i g)(W(\eta)-z-i g)^{-1}
\end{aligned}
$$

and the operator $U$ into a shift operator

$$
(U \Psi)(\eta)=\Psi(\gamma \eta), \gamma \eta=\left(\gamma_{1} \eta_{1}, \ldots, \gamma_{d} \eta_{d}\right), \gamma=\left(\exp 2 \pi i \alpha_{1}, \ldots, \exp 2 \pi i \alpha_{d}\right) .
$$

By virtue of the commutation relation

$$
U^{n} \Phi(\eta)=\left(\Phi\left(\gamma^{n} \eta\right) U^{n}, n \in \mathbb{R}, \gamma^{n}=\left(\gamma_{1}^{n}, \ldots, \gamma_{d}^{n}\right),\right.
$$

valid for any operator $\phi$ of multiplication by a bounded function $\phi(\eta)$ in $L_{2}\left(T_{d}\right)$, 
series (2.4) may be transformed also into the form

$$
R=B-2 i g \sum_{n=1}^{\infty} B \prod_{s=0}^{n-1} C\left(\gamma^{s} \eta\right) B\left(\gamma^{n} \eta\right) V^{n} e^{2 i \omega n}
$$

All the analysis below will be based on Eqs. (2.3), (2.4) and (2.11). We shall also use the following general statements.

Let $v(d \lambda)$ be a complex-valued finite measure on $\mathbb{R}$, and functions $a(z)$ and $b(z)$ are specified by equalities

$$
a(z)=\int_{-\infty}^{\infty}|\lambda-z|^{-2} v(d \lambda), \quad b(z)=\int_{-\infty}^{\infty}(\lambda-z)^{-1} v(d \lambda) .
$$

Proposition 2.1. Let $\Delta$ be a union of a finite or countable set of intervals in $\mathbb{R}$ whose ends have zero $v$-measure. Then

$$
\lim _{\varepsilon \downarrow 0} \varepsilon \pi^{-1} \int_{\Delta} a(\lambda+i \varepsilon) d \lambda=v(\Delta) .
$$

In particular, if $A$ is a self-adjoint operator in the Hilbert space $\mathscr{H}$ with the resolvent $R(z)=(A-z)^{-1}$ and resolution of unity $E(d \lambda)$, then for any vector $h \in \mathscr{H}$

$$
\lim _{\varepsilon \downarrow 0} \varepsilon \pi^{-1} \int_{\Delta}\|R(\lambda+i \varepsilon) h\|^{2}=(E(\Delta) h, h) .
$$

Proposition 2.2. Let $v_{\mathrm{ac}}(d \lambda)$ be an absolutely continuous component of the measure $v(d \lambda)$ and $v_{\mathrm{ac}}^{\prime}$ its density. Then for Lebesgue almost all

$$
\lim _{\varepsilon \downarrow 0} \pi^{-1} \operatorname{Im} b(\lambda+i \varepsilon)=v_{\mathrm{ac}}^{\prime}(\lambda)
$$

If the left-hand side limit exists at all points of a certain interval $(\alpha, \beta)$ and is a bounded function, then the measure $v(d \lambda)$ is absolutely continuous on $(\alpha, \beta)$ and its derivative coincides with this limit almost everywhere on $(\alpha, \beta)$.

In particular, if $\mathscr{H}, A, R(z)$ and $E(d \lambda)$ are the same as in Proposition 2.1 and $E_{\mathrm{ac}}(d \lambda)$ is an absolutely continuous component of the operator-valued measure $E(d \lambda)$, then for any $h \in \mathscr{H}$,

$$
\lim _{\varepsilon \downarrow 0} \pi^{-1} \operatorname{Im}(h, R(\lambda+i \varepsilon) h)=\left[\frac{\partial}{\partial \lambda}\left(h, E_{\mathrm{ac}} h\right)\right](\lambda) .
$$

Before going on to proving the results formulated in Sect. 1, let us use the above derived formula to calculate the integrated density of states (normalized eigenvalue distribution function) $N(\lambda)$ of $H_{d}$. Recall that the function is specified as follows:

$$
N(\lambda)=M\left\{\left(E(\lambda) e_{0}, e_{0}\right)\right\}
$$

where $\ell_{2}\left(\mathbb{Z}^{d}\right) \ni e_{0}=\delta_{x, 0}$ and $M\{\cdot\}$ in this case denotes the operation $\pi^{-1} \int_{0}^{\pi} \ldots d \omega$. (As regards the properties of this function and its role in the spectral 
analysis of metrically transitive random operators, the reader may consult, e.g., refs. 8 and 9.)

Theorem 1. The integrated density of states $N(\lambda)$ of the operator $H_{d}$ from (1.1) is absolutely continuous and its derivative $\rho(\lambda) \equiv N(\lambda)$ (density of states) is

$$
\rho(\lambda)=(2 \pi i)^{-d} \int_{T_{d}} \frac{g}{(\lambda-w(\eta))^{2}+g^{2}} \frac{d \eta}{\eta} .
$$

Proof. It follows from Eqs. (2.15) and (2.4) that

$$
\int(\lambda-z)^{-1} N(d \lambda)=M\left\{\left(R(z) e_{0}, e_{0}\right)\right\}=\left(R_{0}(z+i g) e_{0}, e_{0}\right),
$$

where $R_{0}(z)=(w-z)^{-1}$ is the resolvent of the "nonperturbed" operator $W$. Since, according to (2.2), $R_{0}(z+i g)=B$ and the operator $B$ is a difference one, then by going in (2.17) to the limit $\operatorname{Im} z \downarrow 0$ and using Proposition 2.1, we arrive at Eq. (2.16).

Substitute now in Eq. (1.1), for $\alpha_{x}$, random variables mutually independent and uniformly distributed over $[0, \pi)$. In this case the "potential" $q_{x}$ in operator (1.1) is

$$
q_{x}=g \tan \alpha_{x},
$$

and, as is clear, are random variables mutually independent and Cauchy-distributed, i.e. having the density

$$
p_{C}(q)=\frac{g}{\pi\left(q^{2}+g^{2}\right)}
$$

and the corresponding operator is that of the Lloyd model [8]. For such an operator, a formula analogous to (2.4) also establishes a relation similar to Eq. (2.15), in which $M\{\cdot\}$ denotes now a mathematical expectation operation. ${ }^{5}$ As a result we obtain

Corollary. The densities of states corresponding to the operator (1.1)-(1.3) and in the Lloyd model are equal.

This fact was proved in refs. 4 and 14 in another way.

\section{Proof of Theorem 2}

Condition (1.5) is convenient to rewrite as follows:

$$
\left|1-\gamma^{x}\right| \geqq C|x|^{-\beta}, x \in \mathbb{R}^{d}, x \neq 0 .
$$

We shall also need the following

Lemma 3.1. Let $\varepsilon>0$ and

$$
S_{\varepsilon}=\left\{\xi \in \mathbb{C}:|\xi|=1, \exists C_{\xi}>0:\left|\xi-\gamma^{x}\right| \geqq C_{\xi}\left(1+|x|^{-d-\varepsilon}, x \in \mathbb{Z}^{d}\right\} .\right.
$$

Then $S_{\varepsilon}$ has a full Lebesgue measure on the unit circle $S^{1}$.

5 Another method to calculate the density of states in the Lloyd model may be found in ref. 8 
We are proving this simple statement, because similar reasoning will be used below more than once.

Put for any $\delta>0$,

$$
\begin{gathered}
S_{\delta}^{1}=\bigcup_{x \in \mathbb{Z}^{d}}\left\{\xi \in S_{1}:\left|\operatorname{Arg} \xi-\operatorname{Arg} \gamma^{x}\right| \leqq \delta\left(1+|x|^{-d-\varepsilon}\right\}\right. \\
\tilde{S}_{\delta}^{1}=S^{1} \backslash S_{\delta}^{1}, S^{1}=\{|\xi|=1\} .
\end{gathered}
$$

Then

$$
\begin{gathered}
\operatorname{mes} S_{\delta}^{1} \leqq \\
\delta \sum_{x \in \mathbb{Z}^{d}}(1+|x|)^{-d-\varepsilon} \underset{\delta \rightarrow 0}{\longrightarrow} 0, \\
\operatorname{mes} \tilde{S}_{\delta}^{1} \underset{\delta \rightarrow 0}{\rightarrow} 2 \pi .
\end{gathered}
$$

Therefore the set of points of a unit circle

$$
S_{\varepsilon}=\bigcup_{n \geqq 1} \tilde{S}_{1 / n}^{1}
$$

has a full Lebesgue measure and for all $\xi \in S_{\varepsilon},\left|\xi-\gamma^{x}\right| \geqq C \xi(1+|x|)^{-d-\varepsilon}$.

Note also that from the definition of the set $\widetilde{S}_{\delta}^{1}$ it follows that

$$
\left|1-\gamma^{x} \xi\right| \geqq \delta(1+|x|)^{-d-\varepsilon}, \xi \in \widetilde{S}_{\delta}^{1} .
$$

Denote by $T_{d, \rho}$ a polycylindric domain,

$$
T_{d, \rho}=\left\{\eta \in \mathbb{C}^{d}: e^{-\rho} \leqq\left|\eta_{j}\right| \leqq e^{\rho}, j=1, \ldots, d, \rho>0\right\} .
$$

Then, by virtue of condition (1.2), the function $W(\eta)$ specified by equality (2.7) will be analytic in $T_{d, \rho}$. Besides, because of equalities (2.7) and (2.8) and since the function $W(\eta)$ takes on real values on the set $T_{d} \subset T_{d, \rho}$, the functions $B(\eta)$ and $C(\eta)$ will be analytic for a certain positive $\rho$ in $T_{d, \rho}$. On the other hand, a function $\Psi(\eta)$ arbitrary and analytic in the domain $T_{d, \rho}$ may, as is known, be represented therein as a Loran series (2.5) with the coefficients $\psi_{x}$ specified by equalities (2.6). These coefficients exponentially tend to zero when $|x| \rightarrow \infty$ :

$$
0<\rho_{1}<\rho,\left|\psi_{x}\right| \leqq \text { const } \exp \left(-\rho_{1}|x|\right) .
$$

It follows from this inequality, condition (3.1) and Lemma 3.1, that the following is valid

Lemma 3.2. Denote by $\mathscr{F}_{0}$ the set of functions $\varphi(\eta)$ analytic in the polycylinder $T_{d, \rho}$, such that

$$
\varphi_{0}=(2 \pi i)^{-d} \int_{T_{d}} \varphi(\eta) \eta^{-1} d \eta=0
$$

Then the operator $I-U$ is a one-to-one mapping of $\mathscr{F}_{0}$ into itself, and

$$
\left[(I-U)^{-1} \varphi\right]_{0}=0,\left[(I-U)^{-1} \varphi\right]_{x}=\left(1-\gamma^{x}\right)^{-1} \varphi_{x} .
$$

Consider now the function $C(\eta, z)$ specified by equalities (2.8). This function will apparently be an analytic function of its variables $\eta$ and $z$ on the polycylindric domain $T_{d, \rho} \times L_{g}$, where $\rho$ is a certain positive constant and the strip $L_{g}$ is

$$
L_{g}=\{z \in \mathbb{C}:|\operatorname{Im} z| \leqq g / 2\} .
$$


The function $C(\eta, z)$ is continuous up to the boundary of $T_{d \rho} \times L_{g}$ and apparently does not assume in it nonpositive values. Therefore, having chosen in a standard manner a logarithm branch on the plane $\mathbb{C}$ with a cut along the negative semiaxis, we obtain the following function analytic in $T_{d, \rho} \times L_{g}$ :

$$
f(\eta, z)=\ln C(\eta, z) \text {. }
$$

Using Lemma 3.2, solve the following equation for $t$ :

$$
\begin{aligned}
f(\eta, z) & =f_{0}(z)+t(\eta, z)-(U t)(\eta, z), \\
f_{0}(z) & =(2 \pi i)^{-d} \int_{T_{d}} f(\eta, z) \eta^{-1} d \eta .
\end{aligned}
$$

As a result, we have

$$
t(\eta, z)=(I-U)^{-1}\left[f(\eta, z)-f_{0}(z)\right],
$$

where functions $t(\eta, z)$ and $f_{0}(z)$ have the following properties.

Lemma 3.3. The function $f_{0}(z)$ is analytic in $L_{g}$, and

$$
\begin{gathered}
\operatorname{Re} f_{0}(z)<0 \text { if } \operatorname{Im} z>0, \\
\operatorname{Re} f_{0}(z)=0 \text { if } \operatorname{Im} z=0, \\
f_{0}(\lambda)=\operatorname{im}(\lambda) ; \lambda, m(\lambda) \in \mathbb{R}, \\
m(\lambda)=2(2 \pi i)^{-d} \int_{T_{d}} \arctan \left[\frac{W(\eta)-\lambda}{g}\right] \eta^{-1} d \eta .
\end{gathered}
$$

Then function $m(\lambda)$ is monotonic, gives one-to-one correspondence between $\mathbb{R}$ and the interval $(-\pi, \pi)$ and is related to the integrated density of states $N(\lambda)$ of Eqs. (2.15)-(2.16) as follows:

$$
2 \pi N(\lambda)=\pi-m(\lambda)
$$

The function $t(\eta, z)$ is analytic in the domain $T_{d, \rho} \times L_{g}$, and

$$
\operatorname{Re} t(\eta, z)=\operatorname{Re} f(\eta, z)=0, \operatorname{Im} z=0 .
$$

Proof. Equations (3.13) and (3.14) and the properties of functions $f_{0}(z)$ and $m(\lambda)$ follow directly from the representation (3.9), (3.11) of Eq. (2.8) and Theorem 1. Analyticity of $t(\eta, z)$ follows from its representation by equality (3.12) and Lemma 3.2 .

From Eq. (3.12) it immediately follows that $\operatorname{Re} t(\eta, z)=\operatorname{Re}\left[f(\eta, z)-f_{0}(z)\right]$. But, when $\operatorname{Im} z=0$, we apparently have from (3.9) and (2.8) that $\operatorname{Re} f(\eta, z)=0$. Therefore, due to Eq. (3.13), Eq. (3.15) is valid.

From equality (3.10) and commutation relations (2.10) we obtain the following representation important for all the below discussion:

$$
C U=e^{t} e^{f_{0}(z)} U e^{-t}
$$

Hence and from Eq. (2.1),

$$
H_{d}-z I=B^{-1} e^{t}\left[I-e^{f_{0}(z)} \chi U\right] e^{-t}(I+\chi U)^{-1} .
$$


Lemma 3.4. The set of $H_{d}$ eigenvalues coincides with the set of solutions to equations

$$
m(\lambda)+2 \omega+2 \pi(\alpha, x)=0(\bmod 2 \pi), x \in \mathbb{Z}^{d} .
$$

Here for every $x, E q$. (3.18) has exactly one solution $\lambda_{x}$, and when $x_{1} \neq x_{2}, \lambda_{x_{1}} \neq \lambda_{x_{2}}$. The set of $H_{d}$ eigenvalues $\left\{\lambda_{x}, x \in \mathbb{Z}^{d}\right\}$ is everywhere dense in $\mathbb{R}$. There is for every $\lambda_{x}$ a single eigenfunction $u^{(x)}$ corresponding to it:

$$
u^{(x)}(\eta)=(I+x U) e^{t\left(\eta, \lambda_{x}\right)} \eta^{x}, x \in \mathbb{Z}^{d} .
$$

It follows from the analyticity of $t$ that every eigenfunction $u^{(x)}$, after having been represented as an element of the space $\ell_{2}\left(\mathbb{Z}^{d}\right)$, will be exponentially decreasing at infinity.

Proof. From representation (3.17) it follows that the vector $u$ will be an eigenvector of the operator $H_{d}$ corresponding to the eigenvalue $\lambda$ if and only if

$$
\left(I-e^{f_{0}(\lambda)} \varkappa U\right)\left(e^{-t}(I+\varkappa U)^{-1} u\right)=0,
$$

or, if put

$$
v=e^{-t}(I+\chi U)^{-1} u
$$

then Eq. (3.21) may be rewritten as

$$
U v=\varkappa^{-1} e^{-f_{0}(\lambda)} v
$$

Since the operator $U$ has apparently the complete family of eigenvectors $\eta^{x}$, $x \in \mathbb{Z}^{d}$, then it follows from Eq. (3.22) that all $v$ 's satisfying Eq. (3.22) are exhausted by the set of vectors $\eta^{x}, x \in \mathbb{Z}^{d}$, and the corresponding eigenvalue $\lambda_{x}$ is the solution to Eq. (3.18). The properties of the set $\left\{\lambda_{x}, x \in \mathbb{Z}^{d}\right\}$ enumerated in the lemma follow directly from the properties of the function $m(\lambda)$ of Lemma 3.3. The expression for the eigenvector is to be obtained from Eq. (3.21) with substituting $v=\eta^{x}$.

To prove Theorem 2 it only remains to show that the system of eigenvectors of the operator $H_{d}$ is complete. We shall use equality (2.13) of Proposition 2.1 and show that for any finite interval $\Delta \subset \mathbb{R}$ whose ends do not belong to the set $\left\{\lambda_{x}, x \in \mathbb{Z}^{d}\right\}$ there is a sequence of imbedded intervals $\Delta_{\delta}, \delta>0$, such that each $\Delta_{\delta}$ is a union of a countable number of intervals in $\mathbb{R}$ and

$$
\Delta \supset \Delta_{\delta} \supset \Delta \cap\left\{\lambda_{x}, x \in \mathbb{Z}^{d}\right\} ; \Delta_{\delta} \downarrow \Delta \cap\left\{\lambda_{x}, x \in \mathbb{Z}^{d}\right\}, \delta \downarrow 0, E\left(\Delta_{\delta}\right)=E(\Delta), \delta>0 .
$$

For this we shall need the following auxiliary statement which is a direct corollary of the analyticity of the function $f_{0}$ in the strip $L_{g}$ and relation (3.13).

Lemma 3.5. Let $\Delta$ be an arbitrary interval in $\mathbb{R}$. Then there is a positive $\varepsilon_{0}$ such that for $0 \leqq \varepsilon \leqq \varepsilon_{0}, \lambda \in \Delta$ and $\xi \in S^{1}$, the inequality

$$
\left|e^{f_{0}(\lambda+i \varepsilon)} \xi-1\right| \geqq \frac{1}{2}\left|e^{f_{0}(\lambda)} \xi-1\right|,
$$

is valid.

Proof. Let us take an arbitrary finite interval $\Delta$ in $\mathbb{R}$ whose ends do not belong to the set of eigenvalues $\left\{\lambda_{x}, x \in \mathbb{Z}^{d}\right\}$. Consider the image of $\Delta$ under the mapping $h: \lambda \rightarrow x \exp \left[f_{0}(\lambda)\right]=x \exp [\operatorname{im}(\lambda)]$ and denote it by $h \Delta$, with $h \Delta \subset S^{1}$. As was shown in Lemma 3.3, hA is a diffeomorphism of $\mathbb{R}$ on $S^{1} \backslash\{-1\}$, and it follows 
from equality (3.18) that $h\left(\lambda_{x}\right)=\gamma^{x}$. Take an arbitrary $\delta>0$ and consider sets $\Delta_{\delta}=\Delta \cap h^{-1} S_{\delta}^{1}$ and $\tilde{\Delta}_{\delta}=\Delta \cap h^{-1} \tilde{S}_{\delta}^{1}$ where $S_{\delta}^{1}$ and $\tilde{S}_{\delta}^{1}$ are specified by (3.3). Here apparently,

$$
\Delta=\Delta_{\delta} \cup \tilde{\Delta}_{\delta}, \Delta_{\delta} \cap \tilde{\Delta}_{\delta}=\phi ; \Delta_{\delta} \downarrow \Delta \cap\left\{\lambda_{x}, x \in \mathbb{Z}^{d}\right\}, \delta \downarrow 0
$$

Lemma 3.6. There exists such positive $\varepsilon_{0}$ that when $\delta>0,0 \leqq \varepsilon \leqq \varepsilon_{0}$ and $\lambda \in \tilde{\Delta}_{\delta}$, for a certain positive constant $C_{y, \delta}$ depending on $\delta$ and $y \in \mathbb{Z}^{d}$, the following inequality is valid:

$$
\left\|R(\lambda+i \varepsilon) \eta^{y}\right\| \leqq C_{y, \delta} .
$$

Proof. Indeed, we have from (3.25):

$$
R(z)=(I+\chi U) e^{t}\left[I-e^{f_{0}(z)} \chi U\right]^{-1} e^{-t} B .
$$

Take an arbitrary $y \in \mathbb{Z}^{d}$, consider the vector $R(z) \eta^{y}$, and denote

$$
\Psi(\eta, z)=e^{-t(\eta, z)} B(\eta, z) \eta^{y} .
$$

Owing to analyticity of functions $t(\eta, z)$ and $B(\eta, z)$ in the domain $T_{d, \rho} \times L_{g}$, there exist such positive $p_{1}<p$ and $k$ depending on $\Delta$ that for $0 \leqq \varepsilon \leqq g / 4$ and $\lambda \in \Delta$,

$$
\begin{gathered}
\left|\psi_{x}(\lambda+i \varepsilon)\right| \leqq k \exp \left[-\rho_{1}|x|\right], \\
\left\|(I+\varkappa U) e^{t}\right\| \leqq k .
\end{gathered}
$$

Thus, it follows from (3.26) and (3.29) that the proof of (3.25) reduces to the proof of inequality

$$
\left\|\left(I-e^{f_{0}(\lambda+i \varepsilon)} \varkappa U\right)^{-1} \Psi(\eta, \lambda+i \varepsilon)\right\| \leqq C_{\delta},
$$

where $C_{\delta}$ is a certain positive constant dependent on $\delta$. Let now be the same as in Lemma 3.5 and $\varepsilon_{0} \leqq g / 4$. From $\lambda \in \tilde{\Delta}_{\delta}$ it follows that

$$
e^{f_{0}(\lambda)} x \in \tilde{S}_{\delta}^{1} \text {. }
$$

Using inequality (3.23) of Lemma 3.5 , we obtain for $0 \leqq \varepsilon \leqq \varepsilon_{0}$ and $\lambda \in \tilde{\Delta}_{\delta}$ :

$$
\left|\left[\left(I-e^{f_{0}(\lambda+i \varepsilon)} \chi U\right)^{-1} \Psi\right]_{x}\right|=\left|\left(1-e^{f_{0}(\lambda+i \varepsilon)} \chi \gamma^{x}\right)^{-1} \psi_{x}\right| \leqq 2\left|\left(1-e^{f_{0}(\lambda)} \chi \gamma^{x}\right)\right|\left|\psi_{x}\right| .
$$

Hence as well as from Eqs. (3.31) and (3.6), it follows that

$$
\left|\left[\left(I-e^{f_{0}(\lambda+i \varepsilon)} \chi U\right)^{-1} \Psi\right]_{x}\right| \leqq 2 \delta^{-1}(1+|x|)^{d+\varepsilon}\left|\Psi_{x}\right| .
$$

From inequality (3.28) and inequality (3.32) valid for $0 \leqq \varepsilon \leqq \varepsilon_{0}$ and $\lambda \in \tilde{\Delta}_{\delta}$ follows validity of (3.30) for the same conditions, which completes the proof of the Lemma.

If we now use equality (2.13), then by virtue of Lemma 3.6 we obtain for any $x \in \mathbb{Z}^{d}$ and $\delta>0$ :

$$
\left(E(\Delta) \eta^{x}, \eta^{x}\right)=\left(E\left(\Delta_{\delta}\right) \eta^{x}, \eta^{x}\right) .
$$

Hence and from (3.24) it follows apparently that

$$
E(\Delta)=E\left(\Delta \cap\left\{\lambda_{x}, x \in \mathbb{Z}^{d}\right\}\right) .
$$

The latter equality means completeness of the set of eigenvectors of the operator $H_{d}$, which is the end of the proof to Theorem 2. 
We shall now consider an operator algebra that naturally arises here and includes resolvents of operators of the form of (1.1), as well as its elementary properties. The latter will be helpful in the calculation of the conductivity corresponding to the operators of the form of (1.1) and a certain generalization of Theorem 2.

Let us consider a polycylindric domain

$$
T_{\rho, r}=\left\{(\eta, \xi) \in \mathbb{C}^{d+1}: e^{-\rho} \leqq\left|\eta_{j}\right| \leqq e^{\rho}, j=1, \ldots, d, e^{-r} \leqq|\xi| \leqq e^{r}, \rho, \gamma>0\right\},
$$

and the family $\mathscr{F}_{\rho, r}$ of functions $u(\eta, \xi)$ which are analytic in $T_{\rho, r}$. Such functions may be represented by the Loran series:

$$
\begin{gathered}
u(\eta, \xi)=\sum_{x \in \mathbb{Z}^{d}, k \in \mathbb{Z}} u_{x, k} \eta^{x} \xi^{k}, \\
u_{x, k}=(2 \pi i)^{-d-1} \int_{\left|\eta_{J}\right|=|\xi|=1} u(\eta, \xi) \eta^{-x-1} \xi^{-k-1} d \eta d \xi, \\
\left|u_{x, k}\right| \leqq \text { Const } e^{-\rho_{1}|x|-r_{1}|k|}, 0<\rho_{1}<\rho, 0<r_{1}<r .
\end{gathered}
$$

The function $u \in \mathscr{F}_{\rho, r}$ induces the following operator:

$$
u(\eta, U)=\sum_{x \in \mathbb{Z}^{d}, k \in \mathbb{Z}} u_{x, k} \eta^{x} U^{k}
$$

As the operators $\eta$ and $U$ do not commute, their order in equality (3.37) is important. We shall refer to the function $u_{x, k}, x \in \mathbb{Z}^{d}, k \in \mathbb{Z}$ as the kernel of an operator and denote the family of operators specified by (3.37) for $u \in \mathscr{F}_{\rho, r}$ by $\mathscr{A}_{\rho, r} \cdot \mathscr{A}_{\rho, r}$ will apparently be an algebra; by virtue of the commutation equation $(2.10)$ and the equality $U \eta^{x}=\gamma^{x} \eta^{x}$, we shall have

$$
\begin{gathered}
(u+v)_{x, k}=u_{x, k}+v_{x, k}, \\
(u v)_{x, k}=\sum_{x_{1} \in \mathbb{Z}^{d}, k_{1} \in \mathbb{Z}} u_{x-x_{1}, k-k_{1}} \gamma^{\left(k-k_{1}\right) x_{1}} .
\end{gathered}
$$

If the components of the vector $\alpha$ are rationally independent (for $d=1, \alpha$ is an irrational number), then one readily makes sure that

$$
u(\eta, U)=0 \Leftrightarrow u_{x, k}=0, x \in \mathbb{Z}^{d}, k \in \mathbb{Z} .
$$

Because we shall not be interested in specific $\rho$ and $r$ values, we shall consider the algebra $\mathscr{A}=\underset{\rho, r>0}{U} \mathscr{A}_{\rho, r}$

With every operator $u \in \mathscr{A}$, the following family of operators may be associated:

$$
u_{\omega}=u\left(\eta, e^{2 i \omega} U\right)=\sum_{x, k} u_{x, k} \eta^{x}\left(e^{2 i \omega} U\right)^{k},
$$

or, if, as before, put $\varkappa=e^{2 i \omega}$,

$$
u_{\varkappa}=u(\eta, \varkappa U)=\sum_{x, k} u_{x, k} \eta^{x}(\varkappa U)^{k}
$$

With each such family we shall associate the following averages: 


$$
\begin{gathered}
\left\langle u_{\omega}\right\rangle=\pi^{-1} \int_{0}^{\pi} u_{\omega} d \omega=(2 \pi i)^{-1} \int_{|x|=1} u_{\varkappa} x^{-1} d x, \\
\left\langle u_{\omega}\right\rangle_{0}=\left(\eta^{0},\left\langle u_{\omega}\right\rangle \eta^{0}\right) .
\end{gathered}
$$

Obviously,

$$
\left\langle u_{\omega}\right\rangle=\sum_{x \in \mathbb{Z}^{d}} u_{x, 0} \eta^{x},\left\langle u_{\omega}\right\rangle_{0}=u_{0,0} .
$$

Since families $u_{\omega}$ and $u_{\varkappa}$ are unambiguously specified by the operator $U$, parameters $\omega$ and $x$ will be often omitted from symbols $\left\langle u_{\omega}\right\rangle,\left\langle u_{\omega}\right\rangle_{0}$.

Lemma 3.7. Let $\Psi_{1}(\eta), \Psi_{2}(\eta), \varphi(\xi) \in \mathscr{F}_{\rho, r}$. Then

$$
\begin{gathered}
\left\langle\Psi_{1}(\eta) \varphi(U) \Psi_{2}(\eta)\right\rangle=\Psi_{1}(\eta) \varphi_{0} \Psi_{2}(\eta), \\
\varphi_{0}=(2 \pi i)^{-1} \int_{|x|=1} \varphi(x) x^{-1} d x, \\
\forall x_{1}, x_{2} \in \mathbb{Z}^{d}, k_{1}, k_{2} \in \mathbb{Z}, \\
\left\langle\eta^{x_{1}} U^{k_{1}} \eta^{x_{2}} U^{k_{2}}\right\rangle=\gamma^{k_{1} x_{2}} \delta_{x_{1}+x_{2}} \delta_{k_{1}+k_{2}},
\end{gathered}
$$

where $\delta_{0}=1$ and for $\ell \neq 0, \delta_{\ell}=0$. If $u_{1}, \ldots, u_{n} \in \mathscr{A}$, then

$$
\left\langle u_{1} u_{2} \ldots u_{n}\right\rangle_{0}=\left\langle u_{2} \ldots u_{n} u_{1}\right\rangle_{0} .
$$

Proof. Equations (3.46) and (3.47) naturally follow from the respective definitions, and Eq. (3.48) readily follows from (3.47).

Theorem 2'. Let $V(z), z \in \mathbb{C}$, be a function analytic in the neighbourhood of the unit circle $S^{1}$ and take on real values on $S^{1}$. Then there exists such $\delta_{0}>0$ that for all $\delta \in \mathbb{R},|\delta| \leqq \delta_{0}$, the statements of Theorem 2 hold for the operator

$$
\tilde{H}_{d}=H_{d}+\delta V[\exp (2 \pi i(\alpha, x)+2 i \omega)] \text {. }
$$

To prove this theorem, let us show that the unitary operator mapping the basis $\eta^{x}, x \in \mathbb{Z}^{d}$, into the eigenfunctions $u^{(x)}, x \in \mathbb{Z}^{d}$ of the operator $H_{d}$ belongs to the operator algebra $\mathscr{A}$.

Make the following change of variables:

$$
\lambda=-i \frac{1-\xi}{1+\xi}=b(\xi), \quad|\xi|=1
$$

Then, as follows from the proof of Lemma 3.4, the $\xi_{x}$ values corresponding to the $\lambda_{x}$ eigenvalue may be found from the relation

$$
e^{-f_{0}(b(\xi))}=x \gamma^{x}, \quad x \in \mathbb{Z}^{d}
$$

Notice now that the function $f_{0}(z)$ specified by equalities (3.9) and (3.11) is analytic in the strip $|\operatorname{Im} z| \leqq g / 2$ and the point $z=\infty$ is the branching point; at the same time, as follows from equalities (3.9) and (3.14), the difference between branches of the function is proportional to $2 \pi i$. Therefore the function $\exp \left[-f_{0}(b(\xi))\right]$ is analytic in the neighbourhood of the circumference $|\xi|=1$ and, in view of 
equalities (3.14), it is a one-to-one mapping of the circumference $|\xi|=1$ onto itself, and its inverse function

$$
a=\left[e^{-f_{0}(b(\cdot))}\right]^{-1}
$$

is also analytic and by virtue of (3.14), for certain positive constants $C$ and $r$,

$$
\left|a\left(\xi_{1}\right)-a\left(\xi_{2}\right)\right| \geqq C\left|\xi_{2}-\xi_{1}\right|, e^{-r} \leqq\left|\xi_{1,2}\right| \leqq e^{r} .
$$

From (3.49) and (3.50) we have:

$$
\xi_{x}=a\left(x \gamma^{x}\right), \quad \lambda_{x}=b\left(a\left(x \gamma^{x}\right)\right)
$$

Besides, note also that the function $\exp [t(\eta, b(\xi))]$ is analytic in $T_{\rho, r}$ for some $\rho, r>0$, which can be proved in the same way as for the function $\exp \left[-f_{0}(b(\xi))\right]$.

From (3.18), (3.19) and (3.11), making use of the commutation relations (2.10), obtain

$$
u^{(x)}(\eta)=-2 i g e^{t\left(\eta, \lambda_{x}\right)} B\left(\eta, \lambda_{x}\right) \eta^{x}
$$

Now if we normalize the function $u^{(x)}(\eta)$ to unity, then in view of equalities (3.14), the corresponding normalized eigenfunction $\tilde{u}^{(x)}(\eta)$ of the operator $H_{d}$ is

$$
\begin{gathered}
\tilde{u}^{(x)}(\eta)=e^{t\left(\eta, \lambda_{x}\right)} \tilde{B}\left(\eta, \lambda_{x}\right) \eta^{x} \\
\widetilde{B}(\eta ; \lambda)=\exp [i \arctan \lambda] B(\eta, \lambda) \times\left[(2 \pi i)^{-d} \int_{T_{d}}|B(\eta, \lambda)|^{2} \eta^{-1} d \eta\right]^{-1 / 2}
\end{gathered}
$$

Use representation (2.7) for the function $B(\eta, z)$, then the function $\widetilde{B}(\eta, z)$ is readily shown to be analytic in $T_{\rho, r}$, for some $\rho, r>0$.

Thus, it follows from equalities (3.53) and (3.55), that

$$
\begin{gathered}
\tilde{u}^{(x)}(\eta)=s\left(\eta, x \gamma^{x}\right) \eta^{x}, \\
s(\eta, \cdot)=\left.e^{t(\eta, b(\xi))} \tilde{B}(\eta, b(\xi))\right|_{\xi=a(\cdot)},
\end{gathered}
$$

where the function $s \in \mathscr{F}_{\rho, r}$. In view of commutation relations (2.10), equality (3.57) may be written as

$$
\tilde{u}^{(x)}=s(\eta, U) \eta^{x}
$$

where the unitary operator $s \in \mathscr{A}$.

Since apparently, if $u \in \mathscr{A}$, then $u^{*} \in \mathscr{A}$ too, then $s^{-1}=s^{*} \in \mathscr{A}$. Thus, from (3.49), (3.53) and (3.57) it follows that

$$
s^{-1} H_{d} s=b \circ a(\varkappa U),
$$

where the function $b \circ a(\cdot)$ is meromorphic in the annulus $K_{r}: e^{-r} \leqq|\xi| \leqq e^{r}, r>0$ and maps the circumference $|\xi|=1$ into $\mathbb{R}$. Consider now the family $\mathscr{V}$ similar to that of ref. 3, which consists of the function $V$ meromorphic in the annulus $K_{r}$ for some $r>0$ and possessing the property

$$
\left|V\left(\xi_{1}\right)-V\left(\xi_{2}\right)\right| \geqq C\left|\xi_{1}-\xi_{2}\right|, \xi_{1}, \xi_{2} \in K_{r},
$$

where $C$ is a $V$-dependent positive constant. Then, by virtue of Eqs. (3.49) and (3.52), $b \circ a \in \mathscr{V}$ and, according to ref. 3, there is $\delta_{0}>0$ such that for any $\delta:|\delta| \leqq \delta_{0}$ 
there exists a unitary operator $U_{\delta} \in \mathscr{A}$ such that

$$
U_{\delta}^{-1}\left[b \circ a(\varkappa U)+\delta s^{-1} V s\right] U_{\delta}=V_{1}(\varkappa U),
$$

where $V_{1} \in \mathscr{V}$. Thus,

$$
(s U \delta)^{-1}(H+\delta V) s U_{\delta}=V_{1}(x U),
$$

whence apparently follows the validity of Theorem 2 '.

Remark. This theorem may be proved otherwise as well. Indeed, as follows from ref. 9, the spectrum of operator (1.1) for almost all $\omega$ 's (in the sense of Lebesgue) will be pure point, if for any interval

$$
\sum_{x \in \mathbb{Z}^{d}} p_{x}(\Delta)=N(\Delta)
$$

where

$$
p_{x}(\Delta)=\lim _{\varepsilon \downarrow 0} \int_{\Delta} d \lambda \varepsilon \pi^{-1} M\left\{\left|R_{0, x}(\lambda+i \varepsilon)\right|^{2}\right\} .
$$

Therefore, one may calculate $p_{x}(\Delta)$, using representations (2.4) and (3.10) and then make sure that Eq. (3.61) is valid.

\section{Upper Bound for Zero Temperature Conductivity}

In this section, by way of avoiding cumbersome formulae, we shall consider the one-dimensional case, but all results hold for the multidimensional case without essential changes in reasoning.

Let $z_{1}=\lambda+i \varepsilon, z_{2}=\lambda+v+i \varepsilon, \varepsilon>0, v>0$ and $R_{j}(\omega)=R\left(z_{j}, \omega\right) j=1,2$ be a resolvent of the operator $H_{1}$ from (1.1).

Put

$$
\sigma_{\varepsilon}(v, \lambda)=-v^{-1} \int_{\lambda}^{\lambda+v}\left\langle W^{\prime}\left(R_{1}-R_{1}^{*}\right) W^{\prime}\left(R_{2}-R_{2}^{*}\right)\right\rangle_{0} d \lambda^{\prime}, W^{\prime}(\eta)=2 \pi i \eta \partial W(\eta) / \partial \eta,
$$

where $\langle A(\omega)\rangle_{0}$ for the operator $A(\omega)$ denotes, as in the preceding section, the operation $\pi^{-1} \int_{0}^{\pi} d \omega\left(\eta^{0}, A(\omega) \eta^{0}\right)$. The term "conductivity corresponding to the external field frequency $v$, the Fermi energy $\lambda$ and the zero temperature" will be applied to

$$
\sigma(v, \lambda)=\varlimsup_{\varepsilon \downarrow 0} \sigma_{\varepsilon}(v, \lambda)
$$

This definition proceeds from the Kubo formula [8] obtained in terms of the linear response theory with "averaging" over the energy range of the length $v$ in the neighbourhood of the Fermi level. Besides, the formula omits the factor consisting of the electron charge, the Planck constant and the number $\pi$.

Let us calculate the integrand in (4.1). To do so, consider the following func- 
tions:

$$
\begin{gathered}
B^{ \pm}(\eta, z)=(W(\eta)-z \pm i g)^{-1}, \\
V^{ \pm}(\eta)=W^{\prime}(\eta) B_{1}^{ \pm}(\eta) e^{ \pm t_{1}(\eta)} B_{2}^{ \pm *}(\eta) e^{ \pm t_{2}^{*}}(\eta),
\end{gathered}
$$

where the function $t(\eta, z)$ is specified by (3.12) and the subscript $j$ of the function $t$, as in case of other functions below, means substitution of $z_{j}$ for $z$. Introduce also

$$
\beta_{m}^{ \pm}=(2 \pi i)^{-1} \int_{|\eta|=1} V^{ \pm}(\eta) \eta^{-m-1} d \eta, m \in \mathbb{Z} .
$$

Note that in view of equality $(2.7), B^{-}(\eta, z)=B(\eta, z)$ and here Eq. (2.8) becomes as follows:

$$
C^{ \pm 1}(\eta, z)=-1 \mp 2 i g B^{\mp}(\eta, z) .
$$

Now it appears to be more convenient to use the usual Fourier transform, i.e. to put

$$
\eta=e^{2 \pi i p}, \quad|p| \leqq 1 / 2 \text {. }
$$

With this substitution, the above functions of $\eta,|\eta|=1, W(\eta), B(\eta)$ and others become periodic functions of $p$ with the period 1 , for which we shall use the symbols as before: $W(p), B(p)$, etc.

Lemma 4.1. If the function $f_{0}(z)$ is defined by equality $(3.11), f_{0, j}=f_{0}\left(z_{j}\right)$ and $\gamma=e^{2 \pi i \alpha}$, then for $W^{\prime}(p)=\partial W(p) / \partial p$,

$$
\begin{gathered}
\left\langle W^{\prime}\left(R_{1}-R_{1}^{*}\right) W^{\prime}\left(R_{2}-R_{2}^{*}\right)\right\rangle_{0}=-4(g+\varepsilon)^{2} \int_{-1 / 2}^{1 / 2} d p W^{\prime 2}(p)\left|B_{1} B_{2}\right|^{2} \\
+4 g^{2} \operatorname{Re} \int_{-1 / 2}^{1 / 2} d p W^{\prime 2}(p) B_{1} B_{1}^{+} B_{2} B_{2}^{+}-4 g^{2} \operatorname{Re} \sum_{m \in \mathbb{Z}} \beta_{m}^{-} \beta_{m}^{+} \frac{1+\gamma^{m} e^{f_{0,1}+f^{*}, 2}}{1-\gamma^{m} e^{f_{0,1}+f^{*}, 2}}
\end{gathered}
$$

Proof. First of all, note that from the definition of the mean value of $\langle\cdot\rangle_{0}$ and Eq. (3.48) it follows that

$$
\begin{aligned}
& \left\langle W^{\prime} R_{1}^{*} W^{\prime} R_{2}^{*}\right\rangle_{0}=\left\langle W^{\prime} R_{1} W^{\prime} R_{2}\right\rangle_{0}^{*}, \\
& \left\langle W^{\prime} R_{1} W^{\prime} R_{2}^{*}\right\rangle_{0}=\left\langle W^{\prime} R_{1}^{*} W^{\prime} R_{2}\right\rangle_{0}^{*} .
\end{aligned}
$$

In calculation of these mean values we shall use representation (3.26) for $R(z)$. To be able to use equalities (3.46) of Lemma 3.7, we transform, by commutation relations (2.10), the said representation in the following way:

$$
R=e^{t}\left(I+C^{-1} e^{f_{0}(z)} \chi U\right)\left(I-e^{f_{0}(z)} \chi U\right)^{-1} e^{-t} B .
$$

Representation (4.10) was obtained by using also equality (3.10). Since, in view of (3.13), $R$ is analytic in the circle $|x| \leqq 1$, then, by Lemma 3.7,

$$
\left\langle W^{\prime} R_{1} W^{\prime} R_{2}\right\rangle_{0}=(2 \pi i)^{-1} \int_{|\eta|=1} W^{\prime 2}(\eta) B_{1}(\eta) B_{2}(\eta) \eta^{-1} d \eta
$$

Now use representation (4.10) for calculation of the average $\left\langle W^{\prime} R_{1} W^{\prime} R_{2}\right\rangle_{0}$; 
that is, on the basis of commutation relations (2.10) and the above defined functions $V^{ \pm}$, obtain

$$
\begin{aligned}
W^{\prime} R_{1} W^{\prime} R_{2}= & \sum_{m} \beta_{m}^{-}\left[W^{\prime} e^{t} \eta^{m}\left(I+C_{1}^{-1} e^{f_{0,1}} \gamma^{m} \chi U\right) \times\left(I-e^{f_{0,1}} \gamma^{m} \varkappa U\right)^{-1}\right. \\
& \left.\times\left(I-e^{f_{0,2}^{*} \chi^{-1}} U^{-1}\right)^{-1}\left(I+\chi^{-1} U^{-1} e^{f_{0,2}^{*}} C_{2}^{*-1}\right) e^{t_{2}^{*}}\right] .
\end{aligned}
$$

The above expressions are, as functions of $\varkappa U$, meromorphic in the unit circle with the only pole at point $\varkappa=e^{f_{0,2}^{*}}$. From this fact, Eqs. (3.46), (4.4) and identities (4.6), by simple calculations, we arrive at the following:

$$
\begin{aligned}
\left\langle W^{\prime} R_{1} W^{\prime} R_{2}\right\rangle_{0}= & \sum_{m \in \mathbb{Z}} \beta_{m}^{-}\left\langleW ^ { \prime } e ^ { t _ { 1 } } \eta ^ { m } \left[ I+4 g^{2} B_{1}^{+} B_{2}^{+*}\right.\right. \\
& \left.\left.\cdot \frac{\gamma^{m} \exp \left[f_{0,1}+f_{0,2}^{*}\right]}{1-\gamma^{m} \exp \left[f_{0,1}+f_{0,2}^{*}\right]}\right] e^{t_{2}^{*}}\right\rangle_{0} \\
= & 4 g^{2} \sum_{m \in \mathbb{Z}} \beta_{m}^{-}\left\langle V^{+} \eta^{m}\right\rangle_{0} \frac{\gamma^{m} \exp \left[f_{0,1}+{ }_{0,2}^{*}\right]}{1-\gamma^{m} \exp \left[f_{0,1}+f_{0,2}\right]}+\left\langle W^{\prime 2} B_{1} B_{2}^{*}\right\rangle_{0} \\
= & \int_{1 / 2}^{1 / 2} d p\left(W^{\prime 2} B_{1} B_{2}^{*}-2 g^{2} V^{+} V^{-}\right) \\
& +2 g^{2} \sum_{m \in \mathbb{Z}} \beta_{m}^{-} \beta_{-m}^{+} \frac{1+\gamma^{m} \exp \left[f_{0,1}+f_{0,2}^{*}\right]}{1-\gamma^{m} \exp \left[f_{0,1}+f_{0,2}^{*}\right]} .
\end{aligned}
$$

From equalities (4.4), (4.8), (4.9), (4.11) and (4.12), by elementary transformations, we obtain (4.7). The lemma is proved.

Substituting now (4.7) into (4.1) and (4.2), we note that the first two terms of (4.7) are, by virtue of (4.3), continuous functions of $z$, when $|\operatorname{Im} z| \leqq g / 2$, and therefore, after going to the limit, $\varepsilon \downarrow 0$ will give no contribution to $\sigma(v, \lambda)$. We introduce now the function

$$
\varphi_{v}(z)=-i\left[f_{0}(z)-f_{0}(z+v)\right] .
$$

From Lemma 3.3 and equality (2.15) the following holds:

Lemma 4.2. If $z=\mu \in \mathbb{R}$, then

$$
\varphi_{v}(\mu)=2 \pi \int_{\mu}^{\mu+v} \rho(\tau) d \tau .
$$

Further, with sufficiently small $v>0$, the function $\varphi_{v}(\mu)$ is monotonic for $\mu \in[\lambda, \lambda+v]$, takes on values in the range $\left[\varphi_{v}(\lambda), \varphi_{v}(\lambda+v)\right]$ and the number of solutions to the equation

$$
\varphi_{v}(\mu)=2 \pi m \alpha(\bmod 2 \pi), m \in \mathbb{Z}
$$

in the interval $[\lambda, \lambda+v]$ is 0 or 1 , depending on the validity of relation

$$
2 \pi m \alpha \in\left[\varphi_{v}(\lambda), \varphi_{v}(\lambda+v)\right](\bmod 2 \pi) .
$$

Denote the set of solutions $\mu_{m}$ by $\Lambda_{\lambda, v}$. 
Lemma 4.3. Let $h(\mu)$ be a real-valued continuously differentiable function in the interval $[\lambda, \lambda+v]$. Then, if $\varphi$ is an internal point of the interval $\left[\varphi_{v}(\lambda), \varphi_{v}(\lambda+v)\right]$, i.e. for a some $\mu_{\varphi} \in(\lambda, \lambda+v), \varphi_{v}\left(\mu_{\varphi}\right)=\varphi$, then

$$
\lim _{\varepsilon \downarrow 0} \operatorname{Re} \int_{\lambda}^{\lambda+v} d \mu h(\mu) \frac{1+\exp i\left[\varphi-\varphi_{v}(\mu+i \varepsilon)\right]}{1-\exp i\left[\varphi-\varphi_{v}(\mu+i \varepsilon)\right]}=\frac{2 \pi h\left(\mu_{\varphi}\right)}{\varphi_{v}^{\prime}\left(\mu_{\varphi}\right)} .
$$

If $\varphi$ coincides with one of the ends of the interval $\left[\varphi_{v}(\lambda), \varphi_{v}(\lambda+v)\right]$, then $\mu_{\varphi}$ is equal to $\lambda$ or $\lambda+v$, and we shall have, instead of equality (4.17), the following:

$$
\lim _{\varepsilon \downarrow 0} \operatorname{Re} \int_{\lambda}^{\lambda+v} d \mu h(\mu) \frac{1+\exp i\left[\varphi-\varphi_{v}(\mu+i \varepsilon)\right]}{1-\exp i\left[\varphi-\varphi_{v}(\mu+i \varepsilon)\right]}=\frac{\pi h\left(\mu_{\varphi}\right)}{\varphi_{v}^{\prime}\left(\mu_{\varphi}\right)} .
$$

Proof. Note first of all that from analyticity of the function $\varphi_{v}(z)$, in view of (4.13), and its monotonicity in the interval $[\lambda, \lambda+v]$ by virtue of Lemma 4.2 , it follows that the function

$$
1+\exp i\left[\varphi-\varphi_{v}(z)\right] / 1-\exp i\left[\varphi-\varphi_{v}(z)\right]
$$

is meromorphic and in the domain $\operatorname{Re} z \in[\lambda, \lambda+v],|\operatorname{Im} z|<\varepsilon$ for sufficiently small $\varepsilon$, has its sole pole at the point $z=\mu_{\varphi}$. From this fact, smoothness of $h$ and the obvious fact that function (4.19) assumes imaginary values when $\varphi_{v}(z)$ is real, it follows that

$$
\begin{aligned}
& \lim _{\varepsilon \downarrow 0} \operatorname{Re} \int_{\lambda}^{\lambda+v} d \mu h(\mu) \frac{1+\exp i\left(\varphi-\varphi_{\nu}(\mu+i \varepsilon)\right]}{1-\exp i\left[\varphi-\varphi_{\nu}(\mu+i \varepsilon)\right]} \\
& \quad=\lim _{\varepsilon \downarrow 0} h\left(\mu_{\varphi}\right) \operatorname{Re} \int_{\lambda}^{\lambda+v} d \mu \frac{1+\exp i\left[\varphi-\varphi_{v}(\mu+i \varepsilon)\right]}{1-\exp i\left[\varphi-\varphi_{v}(\mu+i \varepsilon)\right]} .
\end{aligned}
$$

By calculating the right-hand side of this equality using standard methods of complex variable function theory, we obtain the sought Eqs. (4.17) and (4.18). In this case Lemma 4.3 , as is readily seen, admits the following generalization:

Lemma 4.4. If $h(\mu, \varepsilon)$ is a continuously differentiable function of its arguments and the function $h(\mu) \equiv h(\mu, 0)$ is real-valued, then Eqs. (4.17) and (4.18) will remain valid, if $h(\mu)$ is substituted by $h(\mu, \varepsilon)$ as the integrand.

Regarding the coefficients $\beta_{m}^{ \pm}$entering into Eq. (4.7), note that in view of (4.4) and (4.5) and analyticity of the functions $V^{ \pm}(\eta, z)$, when $\eta \in T_{1, \rho}$ and $|\operatorname{Im} z| \leqq g / 2$ the following statement is true.

Lemma 4.5. There exist positive constants $C$ and $\rho$ such that when $\operatorname{Re} z \in[\lambda, \lambda+v]$ and $|\operatorname{Im} z| \leqq g / 2$,

$$
\left|\beta_{m}^{ \pm}(z)\right| \leqq C e^{-\rho|m|}, m \in \mathbb{Z}
$$

Besides, when $z=\mu \in \mathbb{R}$,

$$
\beta_{-m}^{+}(\mu)=\left(\beta_{m}^{-}(\mu)\right)^{*} .
$$

Now calculate the conductivity $\sigma(v, \lambda)$ by Eq. (4.7). Consider the following identity: 


$$
\begin{aligned}
& \beta_{m}^{-} \beta_{-m}^{+} \frac{1+\gamma^{m} \exp \left[f_{0,1}+f_{0,2}^{*}\right]}{1-\gamma^{m} \exp \left[f_{0,1}+f_{0,2}^{*}\right]} \\
& \left.\quad=\beta_{m}^{-} \beta_{-m}^{+} \frac{1+\gamma^{m} \exp \left[f_{0,1}+f_{0,2}^{*}\right]}{1-\gamma^{m} \exp \left[f_{0,1}+f_{0,2}^{*}\right]} \times \frac{1-\gamma^{m} e^{-i \varphi_{v}}}{1+\gamma^{m} e^{-i \varphi_{v}}}\right] \frac{1+\gamma^{m} e^{-i \varphi_{v}}}{1-\gamma^{m} e^{-i \varphi_{v}}}
\end{aligned}
$$

Since by virtue of Lemma 3.3 in the case of $\varepsilon=0 f_{0,1}+f_{0,2}^{*}=-i \varphi_{v}$ then, by using Lemmas 4.3 and 4.4, where the role of the function $h$ will be played by the parenthetical expression in the right-hand side of identity (4.22), and also Lemma 4.5, we obtain from (4.7), (4.1), (4.2) the following:

$$
\sigma(v, \lambda)=\frac{8 \pi}{v} g^{2} \sum_{\mu_{m} \in \Lambda_{\lambda, v}}^{\prime} \frac{\left|\beta_{m}^{-}\left(\mu_{m}\right)\right|^{2}}{\varphi_{v}^{\prime}\left(\mu_{m}\right)}
$$

where $\sum^{\prime}$ means that if one of the ends of the interval $[\lambda, \lambda+v]$ equals $\mu_{m}$, then $1 / 2$ must be placed before the pertinent term. From Eq. (4.23) it apparently follows that

$$
|\sigma(v, \lambda)| \leqq \frac{8 \pi g^{2}}{v} \sum_{\mu_{m} \in \lambda, v} \frac{\left|\beta_{m}^{-}\left(\mu_{m}\right)\right|^{2}}{\left|\varphi_{v}^{\prime}\left(\mu_{m}\right)\right|} .
$$

The asymptotic bound of a simpler form for the conductivity $\sigma(v, \lambda)$ from inequality (4.24) will be obtained by inequalities (4.20) and the following statement.

Lemma 4.6. Let $\mu_{m}, \mu_{m}^{\prime} \in \Lambda_{\lambda, v}$ be different. Then there exists a positive $v$-independent constant $C_{1}$ such that

$$
\left|m-m^{\prime}\right| \geqq C_{1} v^{-1 / \beta},
$$

where $\beta$ is the constant of condition (1.5).

Besides, if $\mu_{m} \in \Lambda_{\lambda, v}$, then

$$
|m| \geqq C_{1} v^{-1 / \beta} .
$$

Proof. Note first of all that condition (1.5) may be rewritten as Eq. (3.1), namely:

$$
\left|e^{2 \pi i m \alpha}-1\right| \geqq C|m|^{-\beta} \text {. }
$$

Besides, by virtue of (4.14) it is apparent that

$$
\max _{\mu, \mu^{\prime} \in[\lambda, \lambda+v]}\left|\varphi_{\nu}(\mu)-\varphi_{\nu}\left(\mu^{\prime}\right)\right| \leqq 2 v \max _{\mu \in[\lambda, \lambda+v]} \rho(\mu) .
$$

Because $\mu_{m}$ and $\mu_{m}^{\prime}$ are solutions to Eq. (4.15) in $[\lambda, \lambda+v]$ then $2 \pi\left(m-m^{\prime}\right)=\varphi_{v}\left(\mu_{m}\right)$ $-\varphi_{v}\left(\mu_{m^{\prime}}\right)(\bmod 2 \mathrm{~A})$. Hence, in view of Eqs. (4.27) and (4.28), obtain for a certain constant $\tilde{C}$ independent of $v$ :

$$
C\left|m-m^{\prime}\right|^{-\beta} \leqq\left|e^{2 \pi i\left(m-m^{\prime}\right) \alpha}-1\right|=\left|\exp i\left[\varphi_{v}\left(\mu_{m}\right)-\varphi_{v}\left(\mu_{m^{\prime}}\right)\right]-1\right| \leqq \widetilde{C} v .
$$

From the latter inequality easily follows validity of inequality (4.25).

For proving inequality (4.26) note that, in view of (4.14) for $0 \leqq v \leqq 1$ and some positive-independent constants $\widetilde{C}_{1}$ and $\widetilde{C}_{2}$,

$$
\widetilde{C}_{2} v \geqq\left|\varphi_{v}(\mu)\right| \geqq \widetilde{C}_{1} v, \quad \mu \in[\lambda, \lambda+v] .
$$


Since $\mu_{m}$ is the solution of Eq. (4.15), then

$$
\varphi_{v}\left(\mu_{m}\right)=2 \pi m \alpha(\bmod 2 \pi)
$$

Hence, and from Eqs. (4.27), (4.29), we obtain that

$$
C|m|^{-\beta} \leqq\left|e^{2 \pi i m \alpha}-1\right|=\left|e^{i \varphi_{v}\left(\mu_{m}\right)}-1\right| \leqq C_{3} v,
$$

where $C_{3}$ is the positive $v$-independent constant. From the latter inequality apparently follows the sought inequality (4.26).

From inequalities (4.20), (4.25), (4.26) and (4.24) it follows, as is easily seen, validity of the asymptotic inequality (1.6), which is the conclusion of the proof of Theorem 3.

\section{Appendix: Proof of Theorem 4}

If an irrational $\alpha$ is such that for any integer $r$ and $q$,

$$
|\alpha-r / q| \geqq q^{-20},
$$

then by virtue of Theorem 2 the spectrum of the operator $H_{1}$ will be pure point, i.e. the absolutely continuous component of the spectrum is absent (we took 20 in (A.1) only to avoid fractional degrees of $q$ below). Assume now that condition (A.1) is not fulfilled, i.e. there is a sequence of integers $r_{n}$ and natural $q_{n}$, where $q_{n}$ are mutually prime, that when $n \rightarrow \infty, r_{n} / q_{n} \rightarrow \alpha$, and

$$
\left|\alpha-r_{n} / q_{n}\right| \leqq q_{n}^{-20} \text {. }
$$

Show now that for any $\omega$ satisfying condition (1.4) and $\alpha$ satisfying (A.2), the absolutely continuous component of the spectrum of the operator $H_{1}$ is absent. To do so, it is by Proposition 2.2 sufficient to ascertain that for each fixed $x \in \mathbb{Z}$, for Lebesgue almost all $\lambda$ 's, the following relation is valid:

$$
\lim _{\varepsilon \downarrow 0} \operatorname{Im}\left(\eta^{x}, R(\lambda+i \varepsilon) \eta^{x}\right)=0,
$$

where for the resolvent $R$, its representation in the space $L_{2}\left(T_{1}\right)=L_{2}\left(S^{1}\right)$ should be taken. The proof below of (A.3) is arranged as follows. The operator $H_{1}$ and its resolvent $R$ depend on $\alpha$, i.e. $R(\lambda+i \varepsilon)=R(\lambda+i \varepsilon, \alpha)$. Since, in view of (A.2), $\alpha$ is sufficiently well approximable by rational numbers, it is possible, by using the representation of $R$ as series (2.11), to reduce the proof of (A.3) to establishment of validity of the following relation:

$$
\lim _{n \rightarrow \infty} \operatorname{Im}\left(\eta^{x}, R\left(\lambda+i \varepsilon_{n}, r_{n} / q_{n}\right) \eta^{x}\right)=0, x \in \mathbb{Z},
$$

where $\varepsilon_{n}=q_{n}^{-5}$, for Lebesgue almost all $\lambda$ 's. This is proved with the use of suitable estimates of the expression under the limit sign, which can be obtained on the basis of the said representation (2.4).

In this Appendix, as in the preceding sections, it is convenient to consider the operator representations in the space $L_{2}\left(S^{1}\right)$; in particular here it is convenient, as in Sect. 4, to substitute $\eta$ by $p$ :

$$
S^{1} \ni \eta=e^{2 \pi i p}, p \in \mathbb{R} .
$$


As a result, Eq. (2.10) becomes

$$
(U \Psi)(p)=\Psi(p+\alpha), U^{n} \Phi(p)=\Phi(p+n \alpha) U^{n}, n \in \mathbb{Z} .
$$

Hence, and from (2.4) we obtain

$$
\begin{gathered}
R(z)=\sum_{n=0}^{\infty} R_{n}(p) U^{n} \varkappa^{n}, \\
R_{0}(p)=B(p), R_{n}(p)=-2 i g B(p) \prod_{s=0}^{n-1} C(p+s \alpha) B(p+n \alpha) .
\end{gathered}
$$

Now fix the arbitrary finite interval $\Delta$ and introduce:

$$
L_{\Delta}=\{z \in \mathbb{C}: \operatorname{Re} z \in \Delta, 0 \leqq \operatorname{Im} z \leqq g / 4\} .
$$

From the definitions of functions $B$ and $C$ by equalities (2.7) and (2.8), we obtain the following estimates valid for:

$$
\begin{gathered}
|B(p, \lambda+i \varepsilon)| \leqq C_{1}, \\
|C(p, \lambda+i \varepsilon)| \leqq e^{-C_{2} \varepsilon} \\
\left|R_{n}(p, \lambda+i \varepsilon)\right| \leqq C_{1} e^{-C_{2} n \varepsilon} \\
\left|\frac{B\left(p+p_{1}\right)}{C(p)}-1\right| \leqq C_{1}\left|p_{1}\right|, \\
\left|\frac{C\left(p+p_{1}\right)}{C(p)}-1\right| \leqq C_{1}\left|p_{1}\right|,
\end{gathered}
$$

where $C_{1}$ and $C_{2}$ are positive constants independent of $z \in L_{\Delta}$ and $\alpha$.

Lemma A.1. Let $\alpha_{1}, \alpha_{2} \in \mathbb{R}, z=\lambda+i \varepsilon \in L_{\Delta}$. Then there exist positive constants $C_{3}$ and $C_{4}$ independent of $z \in L_{\Delta}$ and such that for any positive integer $k$,

$$
\begin{aligned}
& \left|\left(\eta^{x}, R\left(\lambda+i \varepsilon, \alpha_{1}\right) \eta^{x}\right)-\left(\eta^{x}, R\left(\lambda+i \varepsilon, \alpha_{2}\right) \eta^{x}\right)\right| \\
& \quad \leqq C_{3}\left|\alpha_{1}-\alpha_{2}\right|^{1 / 2} \int_{0}^{C_{3} k\left|\alpha_{1}-\alpha_{2}\right|^{1 / 2}} d \tau\left(e^{\tau^{2}}-1\right)+C_{3} \varepsilon^{-1} e^{-C_{4} k \varepsilon} .
\end{aligned}
$$

Proof. It follows from Eq. (A.7) that

$$
\begin{aligned}
& R(z, \alpha) \eta^{x}=\sum_{n=0}^{\infty} \widetilde{R}_{n, x}(p, \alpha) \eta^{x}, \\
& \widetilde{R}_{n, x}(p, \alpha)=R_{n}(p, \alpha)\left(x e^{2 \pi i \alpha x}\right)^{n},
\end{aligned}
$$

and from the definition of $R_{n}(p, \alpha)$ by equalities (A.7) and inequalities (A.9)-(A.13) we have for $n \geqq 0$ and $\Delta \alpha \equiv\left|\alpha_{2}-\alpha_{1}\right|$.

$$
\begin{aligned}
& \left|\frac{\tilde{R}_{n, x}\left(p, \alpha_{2}\right)}{\tilde{R}_{n, x}\left(p, \alpha_{1}\right)}-1\right| \leqq 2 \pi \Delta \alpha n|x| \\
& \quad+\left(1+n C_{1} \Delta \alpha\right) \prod_{s=1}^{n}\left(1+C_{1} s \Delta \alpha\right)-1 \leqq 2 \pi \Delta \alpha n|x|
\end{aligned}
$$




$$
+\exp \left[C_{1} n \Delta \alpha+\sum_{s=1}^{n} C_{1} s \Delta \eta\right]-1 \leqq \exp \left[C_{1}^{\prime} \Delta \alpha n^{2}\right]-1 .
$$

From (A.11) and (A.17), in view of the identity

$$
\tilde{R}_{n, x}\left(p, \alpha_{2}\right)-\tilde{R}_{n, x}\left(p, \alpha_{1}\right)=\left(\frac{\tilde{R}_{n, x}\left(p, \alpha_{2}\right)}{\tilde{R}_{n, x}\left(p, \alpha_{1}\right)}-1\right) \tilde{R}_{n, x}\left(p, \alpha_{1}\right),
$$

we obtain with allowance for (A.7) and (A.15), (A.16):

$$
\begin{aligned}
& \left|\left(\eta^{x}, R\left(\lambda+i \varepsilon, \alpha_{1}\right) \eta^{x}\right)-\left(\eta^{x}, R\left(\lambda+i \varepsilon, \alpha_{2}\right) \eta^{x}\right)\right| \\
& \quad \leqq \sum_{n=0}^{\infty} \int_{-1 / 2}^{1 / 2} d p\left|\widetilde{R}_{n, x}\left(p, \alpha_{2}\right)-\widetilde{R}_{n, x}\left(p, \alpha_{1}\right)\right| \\
& \quad \leqq \sum_{n=0}^{k-1}\left(e^{C^{\prime} \Delta \Delta n^{2}}-1\right) C_{1} e^{-C_{2} n \varepsilon}+\sum_{n=k}^{\infty} 2 C_{1} e^{-C_{2} n \varepsilon} \\
& \quad \leqq C_{1} \int_{0}^{k} d \tau\left(e^{C_{1}^{\prime} \Delta \alpha \tau^{2}}-1\right)+2 C_{1} e^{-C_{2} k \varepsilon}\left(1-e^{-C_{2} \varepsilon}\right)^{-1} .
\end{aligned}
$$

From this estimate follows inequality (A.14).

Note now that the validity of the limiting relation (A.3) is enough to be established for any sequence $\varepsilon_{n}$ converging to zero. Choose it in the following way. Consider the sequence $r_{n} / q_{n}$ approximating the number $\alpha$ with the rate specified by inequalities (A.2) and put

$$
\varepsilon_{n}=q_{n}^{-5}
$$

Now use Lemma A.1, putting $\alpha_{1}=\alpha, \alpha_{2}=r_{n} / q_{n}$ and substituting

$$
k_{n}=q_{n}^{6}
$$

for $k$. As the result we obtain the following statement:

Lemma A.2. For any $\lambda \in \Delta$,

$$
\lim \operatorname{Im}\left(\eta^{x}, R(\lambda+i \varepsilon, \alpha) \eta^{x}\right)=\lim \operatorname{Im}\left(\eta^{x}, R\left(\lambda+i \varepsilon_{n}, r_{n} / q_{n}\right) \eta^{x}\right)
$$

where $R(z, \alpha)$ is specified by equalities $(A .7)$, and $\varepsilon_{n}=q_{n}^{-5}$.

Indeed, in view of (A.20) and (A.2), when $n \rightarrow \infty, k_{n}\left|\alpha-r_{n} q_{n}\right|^{1 / 2} \rightarrow 0$. Hence and from inequality (A.14), we obtain for $n \rightarrow \infty$, with allowance for (A.19), (A.20) and (A.2):

$$
\begin{gathered}
\left|\left(\eta^{x}, R\left(\lambda+i \varepsilon_{n}, \alpha\right) \eta^{x}\right)-\left(\eta^{x}, R\left(\lambda+i \varepsilon_{n}, r_{n} / q_{n}\right) \eta^{x}\right)\right| \\
\leqq 2 C_{3}^{4} k_{n}^{2}\left|\alpha-r_{n} / q_{n}\right|+C_{3} \varepsilon_{n}^{-1} e^{-C_{4} k_{n} \varepsilon_{n}} \rightarrow 0 .
\end{gathered}
$$

Thus, Lemma A. 2 is proved.

Now, for the proof of the theorem, by virtue of relation (A.21), it is enough to ascertain that, when $\varepsilon_{n}=q_{n}^{-5}$, for Lebesgue almost all $\lambda \in \Delta$,

$$
\lim _{n \rightarrow \infty} \operatorname{Im}\left(\eta^{x}, R\left(\lambda+i \varepsilon_{n} ; r_{n} / q_{n}\right) \eta^{x}\right)=0
$$


Investigate $R(z, \alpha)$ specified by (A.7) for rational $\alpha$ 's, i.e. for $\alpha=r / q$, where $r, q$ are integer mutually prime numbers.

Let us consider the following auxiliary functions:

$$
\begin{gathered}
C_{q}(p)=\prod_{s=1}^{q} C\left(p+s r q^{-1}\right) \\
D_{k}(p)=-2 i g B(p) B\left(p+(k+1) r q^{-1}\right) \prod_{s=1}^{k} C\left(p+s r q^{-1}\right) .
\end{gathered}
$$

Then, if we denote

$$
x_{q}=x \exp 2 \pi i r q^{-1} x, x \in \mathbb{Z},
$$

then $x_{q}^{q}=x^{q}$, and for $n \geqq 1$ and $n-1=\ell q+k, 0 \leqq k \leqq q-1$, we shall have

$$
\tilde{C}_{n, x}(p)=\left[C_{q}(p) \varkappa^{q}\right]^{l} \mathscr{D}_{k}(p) \varkappa_{q}^{k+1} \text {. }
$$

Now if we use the identity

$$
\operatorname{Im}\left(\eta^{x}, R(\lambda+i \varepsilon) \eta^{x}\right)=\varepsilon\left\|R(\lambda+i \varepsilon) \eta^{x}\right\|^{2},
$$

then in view of Eqs. (A.15) and (A.16), we shall obtain

$$
\operatorname{Im}\left(\eta^{x}, R(\lambda+i \varepsilon, r / q) \eta^{x}\right)=\varepsilon \int_{-1 / 2}^{1 / 2} d p\left|R_{0}(p)+\sum_{n=1}^{\infty} \tilde{R}_{n, x}(p)\right|^{2} .
$$

If we use inequality (A.11), then by virtue of (A.28) for the proof of (A.22) it is sufficient to ascertain the validity of the following statement:

Lemma A.3. For Lebesgue almost all $\lambda \in \Delta$,

$$
\lim _{n \rightarrow \infty} \varepsilon_{n} \int_{-1 / 2}^{1 / 2} d p\left|\sum_{m=1}^{\infty} \widetilde{R}_{m, x}\left(p, \lambda+i \varepsilon_{n}, r_{n} / q_{n}\right)\right|^{2}=0,
$$

where $\varepsilon_{n}=q_{n}^{-5}$.

Proof. It follows from (A.26) that

$$
\sum_{m=1}^{\infty} \tilde{R}_{m, x}(p)=\left[\sum_{k=0}^{q-1} \mathscr{D}_{k}(p) \varkappa_{q}^{k+1}\right]\left(1-C_{q}(p) x^{q}\right)^{-1} .
$$

Hence, and from Eqs. (A.10) and (A.11) follows existence of such a positive constant $C$ that $\forall z \in L_{\Delta}$

$$
\left|\sum_{n=1}^{\infty} \tilde{R}_{n, x}(p, z, r / q)\right| \leqq C q\left|1-C_{q}(p) x^{q}\right|^{-1} .
$$

Now consider the function $C_{q}(p)$, and note that in view of Eqs. (A.23) and (3.9), it may be represented as follows:

$$
C_{q}(p)=\exp \left[q f_{0}+q \sum_{k \neq 0} f_{k q} e^{2 \pi i k q p}\right] .
$$

Since, as was shown in Sect. 3, the function $f(\eta, z)$ is analytic in the domain 
$T_{1, \rho} \times L_{g}$, then there exists a pair of positive constants $C_{1}$ and $C_{2}$ such that

$$
\left|f_{m}(z)\right| \leqq C_{1} e^{-C_{2}|m|}
$$

Hence, and from equality (A.32) we obtain for:

$$
\left|C_{q}(p, z)-e^{q f_{0}(z)}\right| \leqq C_{3} e^{-C_{4} q}
$$

where $C_{3}$ and $C_{4}$ are positive constants. Now if we use Lemma 3.3, then for $z=$ $\lambda+i \varepsilon \in L_{\Delta}$ and $\varepsilon q \leqq 1$ we shall have:

$$
\left|e^{q f_{0}(\lambda+i \varepsilon)}-e^{i q m(\lambda)}\right| \leqq C_{5} \varepsilon q .
$$

If we put $q=q_{n}$ and $\varepsilon_{n}=q_{n}^{-5}$, then inequalities (A.34) and (A.35) will yield for $z=\lambda+i \varepsilon \in L_{\Delta}$ :

$$
\left|C_{q_{n}}\left(p, \lambda+i \varepsilon_{n}\right)-e^{i q_{n} m(\lambda)}\right| \leqq C_{6} q_{n}^{-4},
$$

where $C_{6}$ is a positive constant.

From inequality (A.36) and $\varkappa=e^{i 2 \omega}$, we have for a positive constant $C_{7}$ and and $z=\lambda+i \varepsilon \in L_{\Delta}$ :

$$
\left|C_{q_{n}}\left(p, \lambda+i \varepsilon_{n}\right) \chi^{q_{n}}-e^{i q_{n}(m(\lambda)+2 \omega)}\right| \leqq C_{7} q_{n}^{-4} .
$$

Now if we use the continuity and monotonicity of $m(\lambda)$, we shall see that for Lebesgue almost all $\lambda \in \mathbb{R}$,

$$
\left|1-e^{i q(m(\lambda)+2 \omega)}\right| \geqq C_{7} q^{-1} .
$$

Hence, and from inequalities (A.37) and (A.31) it follows that for almost all $\lambda \in \Delta$,

$$
\left|\sum_{m=1}^{\infty} \widetilde{R}_{m, x}\left(p, \lambda+i \varepsilon_{n}, r_{n} / q_{n}\right)\right|^{2} \leqq C_{8} q_{n}^{2} .
$$

The latter inequality apparently implies validity of Eq. (A.29).

Equation (A.22) is thus true, which is the conclusion of the proof of Theorem 4.

\section{References}

1. Bellissard, J.: Schrödinger operators with almost periodic potentials: An overview. In: Lecture Notes in Physics, Vol. 153. Berlin, Heidelberg, New York: Springer 1982

2. Bellissard, J., Lima, R., Scoppola, E. : Localization in $v$-dimensional incommensurate structures. Commun. Math. Phys. 88, 465-477 (1983)

3. Craig, W.: Pure point spectrum for discrete almost periodic Schrödinger operators. Commun. Math. Phys. 88, 113-131 (1983)

4. Fishman, S., Grempel, D. R., Prange, R. E.: Localization in an incommensurate potential: An exactly solvable model. Phys. Rev. Lett. 49, 833-836 (1982)

5. Gordon, A. Ya.: On the point spectrum of one-dimensional Schrödinger operator. Usp. Mat. Nauk 31, 257-258 (1976)

6. Kac, I. S. : The spectrum multiplicity of the second order differential operator and the eigenvalue expansion. Izv. Akad. Nauk SSSR, Ser. Mat. 27, 1081-1112 (1963)

7. Levitan, B. M.: Pochti-periodicheslie funktsii (Almost periodic functions). Moscow: GITTL, 1953

8. Lifshitz, I. M., Gredeskul, S. A., Pastur, L. A.: Vvedenie v teoriyu neuporyadochennykh sistem 
(Introduction to the theory of disordered systems). Moscow: Nauka, 1982 (to be published by Wiley \& Sons)

9. Pastur, L. A. : Spectral properties of disordered systems in the one-body approximation. Commun. Math. Phys. 75, 179-196 (1980)

10. Pastur, L. A., Figotin, A. L.: Positivity of the Lyapunov exponent and the absence of absolutely continuous spectrum for the almost-Mathieu equation. J. Math. Phys. 25, 774-777 (1984)

11. Pastur, L. A., Figotin, A. L.: Localization in an incommensurate potential: An exactly solvable multidimensional model. Pis'ma ZhETF 37, 575-577 (1983)

12. Pöschl, J.: Examples of discrete Schrödinger operators with pure point spectrum. Commun. Math. Phys. 88, 447-470 (1983)

13. Simon, B.: Almost periodic Schrödinger operators: A review. Adv. Appl. Math. 3, 463-490 (1982)

14. Simon, B.: Equality of the density of states in a wide class of tight-binding Lorentzian random models. Phys. Rev. B27, 3859-3860 (1983)

15. Thouless, D.: A relation between the density of states and range of localization for one-dimensional random systems. J. Phys. C5, 77-81 (1972)

Communicated by Ya. G. Sinai

Received February 10, 1984 
